\title{
Effect of non-metallic inclusions on the local mechanical behaviour of a G42CrMo4 casting
}

\author{
Sebastian Henschel ${ }^{\mathrm{a}}$ and Lutz Krüger \\ Instiute of Materials Engineering, TU Bergakademie Freiberg, Gustav-Zeuner-Str. 5, 09599 Freiberg, \\ Germany
}

\section{Introduction}

It is well known, that non-metallic inclusions have a detrimental effect on damage evolution and, therefore, on deformability of a metal. The damage mechanisms, namely void initiation, void growth and void coalescence, are strongly influenced by the size and distribution of non-metallic inclusions.

Void initiation is promoted by larger inclusions and a weaker interfacial strength between particle and metallic matrix. Void growth rate is accelerated with increasing void size. Therefore, larger inclusions lead to increased void growth rate. There are at least two possibilities for void coalescence: internal necking and void sheet coalescence. Internal necking is observed in metals with only on size class of a dispersed second phase. In contrast, void sheet coalescence is typically the case, when smaller voids are formed in the ligament between growing voids. These smaller voids are initiated at secondphase dispersions, e. g. carbides.

Models describing the damage evolution assume homogeneous distribution of non-metallic inclusions or already initiated voids with a common mean size. In a previous work, it was shown, that agglomerates or clusters of non-metallic inclusion can deteriorate the deformability of a G42CrMo4+QT significantly. A description of the effect of very few though large non-metallic inclusion clusters in a metallic matrix is, therefore, needed. The aim of this study was to investigate the effect of clusters of non-metallic inclusion on the deformability of a high strength steel.

\section{Materials and methods}

In this study, the cast steel G42CrMo4 (EN 10293, 1.7231) was used, see Table 1.

The casting temperature was $1623^{\circ} \mathrm{C}$. Firstly, the melt flowed through a so-called "contaminating filter" coated with loose alumina particles. Secondly, an actual metal melt filter positioned behind the contaminating filter in the casting gate was used to clean the contaminated melt. The heat treatment consists of austenitising at $840^{\circ} \mathrm{C}$ for $20 \mathrm{~min}$, quenching by a high velocity gas flow (He), and finally tempering at $560^{\circ} \mathrm{C}$ for $1 \mathrm{~h}$ in a $\mathrm{N}_{2}$-atmosphere. Thus, the microstructure consists of a tempered

\footnotetext{
${ }^{a}$ Corresponding author: sebastian.henschel@iwt.tu-freiberg.de
}

This is an Open Access article distributed under the terms of the Creative Commons Attribution License 4.0, which permits unrestricted use, distribution, and reproduction in any medium, provided the original work is properly cited. 


\section{MATEC Web of Conferences}

Table 1. Chemical composition in (wt.\%) of the investigated steel determined by glow discharge optical spectroscopy. Balance is Fe.

\begin{tabular}{|l|l|l|l|l|l|l|l|}
\hline $\mathbf{C}$ & $\mathbf{C r}$ & $\mathbf{M o}$ & $\mathbf{M n}$ & $\mathbf{N i}$ & $\mathbf{S i}$ & $\mathbf{A l}$ & $\mathbf{S}$ \\
\hline 0.41 & 0.97 & 0.25 & 0.79 & 0.22 & 0.53 & 0.09 & 0.007 \\
\hline
\end{tabular}

martensite. Tensile tests were performed at loading rates of $10^{-3} \mathrm{~s}^{-1}$ in a Zwick universal testing machine equipped with a cooling chamber. Particle distributions were determined at cross-sections with the aid of an automated image analysis tool. The non-metallic inclusions were identified by setting a specific grey-scale level.

\section{Results and discussion}

Metallographic investigations showed that small inclusions with diameters up to $5 \mu \mathrm{m}$ dominate the size distribution. Analysing the location of the single inclusions, it is shown, that there is only a small distance between many of these small inclusions. Therefore, a statistical clustering procedure was applied. In that procedure, inclusions with distances below $30 \mu \mathrm{m}$ were combined to one virtual inclusion. Depending on the inclusion location distribution, this process is done several times.

Fracture surfaces reveal a clustering of small $\mathrm{Al}_{2} \mathrm{O}_{3}$ - inclusions. These clusters have a diameter of ca. $100 \mu \mathrm{m}$. Single inclusions within the cluster have a diameter of approximately $2 \mu \mathrm{m}$. Furthermore, it is observed that the fracture process is not only affected by the cluster, but also by shear fracture around the cluster. Therefore, the cluster is not necessarily located in the plane of final fracture. As a consequence, a cluster of about $100 \mu \mathrm{m}$ in diameter can produce a shear fracture surface with a diameter of $500 \mu \mathrm{m}$. In this area, the strain is strongly localised which results in a small global strain. Hence, samples containing such clusters showed a relatively low deformability.

The effect of a low temperature is not necessarily a decrease in deformability until fracture. However, the scatter of deformation characteristics is increasing. Furthermore, deformation characteristics describing localised deformation, namely necking and elongation with necking, show a stronger effect of the testing temperature. Analysing the inclusions cluster size dependency of the deformation characteristics, it is shown, that the largest inclusion strongly affects the necking behaviour and, therefore, the true elongation until fracture. Additionally, samples having more than one inclusion agglomerate exhibit a lower deformability than samples having a comparably large inclusion cluster. However, small agglomerates seem to have a negligible effect when present in combination with a considerably larger cluster. Non-agglomerated inclusions also play a minor role with respect to the deformability. These small inclusions may determine the growth and coalescence of the voids. However, initiation of voids is mainly dependent on the large inclusions, namely agglomerates.

Comparing the observed behaviour with existing models, it is shown, that void growth at single inclusions can be reasonably described by McClintok's model. As mentioned earlier, inclusion agglomerates are not necessarily located in the plane of final fracture and can, therefore, produce a large cone (or cup) on the fracture surface. Thermographic analysis reveals that such a cone is formed well before final fracture. Hence, void coalescence can occur locally and accelerates the remaining void growth.

\section{Conclusions}

Small alumina inclusions are present in the form of agglomerates and affect the deformability in a detrimental way. Strain is localized in shear bands around the inclusion clusters. The deformability is mainly determined by the largest inclusion cluster. Additional inclusion clusters which are considerably smaller than the largest inclusion cluster seem to have a minor effect on the deformability. 
Modelling of fracture as a result of void nucleation, growth and coalescence should not only involve mean microstructural parameters, but also information about heterogeneous features which can dominate the deformation behaviour.

The authors gratefully acknowledge the German Research Foundation (DFG) for supporting the Collaborative Research Center CRC 920, subproject C05. 